CUBO A Mathematical Journal Vol.12, № 03, (1-12). October 2010

\title{
Partial Fractions and $q$-Binomial Determinant Identities
}

\author{
Wenchang CHU \\ Dipartimento di Matematica, Università del Salento, \\ Lecce-Arnesano P. O. Box 193, Lecce 73100, Italy \\ email: chu.wenchang@unile.it \\ ChenYing WANG \\ College of Mathematics and Physics, \\ Nanjing University of Information Science and Technology \\ Nanjing 210044, P. R. China \\ email: wang. chenying@163.com \\ AND \\ WENLONG ZHANG \\ Department of Applied Mathematics, \\ Dalian University of Technology, Dalian 116023, P. R. China \\ email: wenlong.dlut@yahoo.com.cn
}

\begin{abstract}
Partial fraction decomposition method is applied to evaluate a general determinant of shifted factorial fractions, which contains several Gaussian binomial determinant identities.
\end{abstract}




\section{RESUMEN}

El método de descomposición en fracción parciales aplicado para evaluar un determinante general de fracciones factoriales trasladadas, la cual contiene varias identidades determinante binomial Gaussiano.

Key words and phrases: The Cauchy double alternant, Partial fractions, $q$-Binomial coefficients.

Math. Subj. Class.: 15A15, 11C20.

Binomial determinant evaluation plays an important role in combinatorial enumeration, particularly in plane partitions. This paper will establish a very general determinant identity through partial fraction decomposition method. It will be shown to be useful in $q$-binomial determinant evaluations with several interesting known and new formulae being exemplified.

\section{Partial Fraction Decomposition}

For two sequences $\left\{\alpha_{k}, \gamma_{k}\right\}_{k \geq 0}$, define the generalized shifted factorials by

$$
\begin{array}{llll}
(x \mid \alpha)_{0}=1 \quad \text { and } \quad(x \mid \alpha)_{n}=\prod_{k=0}^{n-1}\left(1-x \alpha_{k}\right) & \text { with } \quad n \in \mathbb{N}, \\
(y \mid \gamma)_{0}=1 \quad \text { and } \quad(y \mid \gamma)_{n}=\prod_{k=0}^{n-1}\left(1-y \gamma_{k}\right) & \text { with } \quad n \in \mathbb{N} .
\end{array}
$$

When $\alpha_{k}=\gamma_{k}=q^{k}$ for $k \in \mathbb{N}_{0}$, they will reduce to the usual shifted factorials

$$
(x ; q)_{0}=1 \text { and }(x ; q)_{n}=(1-x)(1-q x) \cdots\left(1-q^{n-1} x\right) \text { with } n \in \mathbb{N} .
$$

For the triangular matrix given by $\alpha=\left[\alpha_{i j}\right]_{0 \leq i \leq j<\infty}$, denote its $j$-th column by $\alpha_{j}=\left(\alpha_{0 j}, \alpha_{1 j}, \alpha_{2 j}\right.$, $\left.\cdots, \alpha_{j j}\right)$. Then the main result may be stated as follows.

Theorem 1 (Generalized Cauchy determinant). Let $\left\{x_{k}\right\}_{k=0}^{n}$ be distinct complex numbers. Then there holds the following determinant identity:

$$
\operatorname{det}_{0 \leq i, j \leq n}\left[\frac{\left(x_{i} \mid \alpha_{j}\right)_{j}}{\left(x_{i} \mid \gamma\right)_{j+1}}\right]=\frac{\prod_{0 \leq i<j \leq n}\left(x_{i}-x_{j}\right)\left(\alpha_{i j}-\gamma_{j}\right)}{\prod_{k=0}^{n}\left(x_{k} \mid \gamma\right)_{n+1}} .
$$

The very special case of this theorem with $\alpha_{i j}=\gamma_{i}$ for $i, j \in \mathbb{N}_{0}$ results in the celebrated Cauchy's double alternant (cf. [6, 7]):

$$
\operatorname{det}_{0 \leq i, j \leq n}\left[\frac{1}{1-x_{i} \gamma_{j}}\right]=\frac{\prod_{0 \leq i<j \leq n}\left(x_{i}-x_{j}\right)\left(\gamma_{i}-\gamma_{j}\right)}{\prod_{0 \leq i, j \leq n}\left(1-x_{i} \gamma_{j}\right)}
$$


Proof. Expanding the rational function in partial fractions, we have

$$
\frac{\left(x_{i} \mid \alpha_{j}\right)_{j}}{\left(x_{i} \mid \gamma\right)_{j+1}}=\frac{\prod_{l=0}^{j-1}\left(1-x_{i} \alpha_{\iota j}\right)}{\prod_{k=0}^{j}\left(1-x_{i} \gamma_{k}\right)}=\sum_{k=0}^{j} \frac{w_{k j}}{1-x_{i} \gamma_{k}}
$$

where the connected coefficients are determined by the following limit relation

$$
w_{k j}=\lim _{x_{i} \rightarrow \frac{1}{\gamma_{k}}}\left(1-x_{i} \gamma_{k}\right) \frac{\left(x_{i} \mid \alpha_{j}\right)_{j}}{\left(x_{i} \mid \gamma\right)_{j+1}}=\frac{\prod_{l=0}^{j-1}\left(\alpha_{\iota j}-\gamma_{k}\right)}{\prod_{l=0: \iota \neq k}^{j}\left(\gamma_{\iota}-\gamma_{k}\right)} .
$$

This leads us to the following determinant factorization

$$
\operatorname{det}_{0 \leq i, j \leq n}\left[\frac{\left(x_{i} \mid \alpha_{j}\right)_{j}}{\left(x_{i} \mid \gamma\right)_{j+1}}\right]=\operatorname{det}_{0 \leq i, k \leq n}\left[\frac{1}{1-x_{i} \gamma_{k}}\right] \times \operatorname{det}_{0 \leq k, j \leq n}\left[w_{k j}\right] .
$$

For the matrix $\left[w_{k j}\right]_{0 \leq k, j \leq n}$ is upper triangular, its determinant is equal to the product of its diagonal entries:

$$
\operatorname{det}_{0 \leq k, j \leq n}\left[w_{k j}\right]=\prod_{j=0}^{n} w_{j j}=\prod_{0 \leq i<j \leq n} \frac{\alpha_{i j}-\gamma_{j}}{\gamma_{i}-\gamma_{j}} .
$$

While the first determinant can be evaluated by Cauchy's double alternant (2). Their combination yields the determinant identity stated in Theorem 1 .

Shifting the $\gamma$-parameters by $\gamma_{k} \rightarrow \gamma_{k-1}$, we may state the determinant identity in Theorem 1 in the following more convenient form.

Proposition 2 (Determinant identity). Let $\left\{x_{k}\right\}_{k=0}^{n}$ be distinct complex numbers. Then there holds the following determinant identity:

$$
\operatorname{det}_{0 \leq i, j \leq n}\left[\frac{\left(x_{i} \mid \alpha_{j}\right)_{j}}{\left(x_{i} \mid \gamma\right)_{j}}\right]=\frac{\prod_{0 \leq i<j \leq n}\left(x_{i}-x_{j}\right)\left(\alpha_{i j}-\gamma_{j-1}\right)}{\prod_{k=0}^{n}\left(x_{k} \mid \gamma\right)_{n}} .
$$

Letting $\alpha_{i j}=p^{i} y_{j}$ and $\gamma_{k}=q^{k}$ further in Proposition 2, we have the identity.

Corollary 3 (Bibasic determinant evaluation formula).

$$
\underset{0 \leq i, j \leq n}{\operatorname{det}}\left[\frac{\left(x_{i} y_{j} ; p\right)_{j}}{\left(x_{i} ; q\right)_{j}}\right]=q^{2\left(\begin{array}{c}
n+1 \\
3
\end{array}\right)} \prod_{0 \leq i<j \leq n}\left(x_{j}-x_{i}\right) \prod_{k=0}^{n} \frac{\left(q^{1-k} y_{k} ; p\right)_{k}}{\left(x_{k} ; q\right)_{n}} .
$$

From this corollary, we can derive numerous $q$-binomial determinant identities.

\section{$2 q$-Binomial Determinant Identities}

Define the Gaussian binomial coefficients by

$$
\left[\begin{array}{l}
x \\
n
\end{array}\right]=\frac{\left(q^{1+x-n} ; q\right)_{n}}{(q ; q)_{n}} \quad \text { where } \quad n \in \mathbb{N}_{0} \quad \text { and } \quad x \in \mathbb{C} .
$$

Applying Corollary 3, we show now ten classes of $q$-binomial determinant identities. 
2.1 Expressing the $q$-binomial coefficient in terms of shifted factorials

$$
\left[\begin{array}{c}
X_{i}-j \\
A
\end{array}\right]=q^{-A j}\left[\begin{array}{c}
X_{i} \\
A
\end{array}\right] \frac{\left(q^{A-X_{i}} ; q\right)_{j}}{\left(q^{-X_{i}} ; q\right)_{j}}
$$

we derive the corresponding determinant formula

$$
\begin{aligned}
\operatorname{det}_{0 \leq i, j \leq n}\left[\left[\begin{array}{c}
X_{i}-j \\
A
\end{array}\right]\right] & =\prod_{0 \leq i<j \leq n}\left(q^{-X_{j}}-q^{-X_{i}}\right)\left(1-q^{1+A+i-j}\right) \\
& \times \frac{q^{2\left(\begin{array}{c}
n+1 \\
3
\end{array}\right)-A\left(\begin{array}{c}
n+1 \\
2
\end{array}\right)}}{(q ; q)_{n}^{n+1}} \prod_{k=0}^{n}\left[\begin{array}{c}
X_{k} \\
A
\end{array}\right]\left[\begin{array}{c}
n-1-X_{k} \\
n
\end{array}\right]^{-1}
\end{aligned}
$$

2.2 Rewriting the $q$-binomial coefficient in terms of shifted factorials

$$
\left[\begin{array}{c}
A \\
X_{i}-j
\end{array}\right]=(-1)^{j} q^{-\left({ }_{2}^{j}\right)+j X_{i}}\left[\begin{array}{c}
A \\
X_{i}
\end{array}\right] \frac{\left(q^{-X_{i}} ; q\right)_{j}}{\left(q^{1+A-X_{i}} ; q\right)_{j}}
$$

we get the corresponding determinant identity

$$
\begin{array}{r}
\operatorname{det}_{0 \leq i, j \leq n}\left[q^{-j X_{i}}\left[\begin{array}{c}
A \\
X_{i}-j
\end{array}\right]\right]=\prod_{0 \leq i<j \leq n}\left(q^{-X_{i}}-q^{-X_{j}}\right)\left(1-q^{-A+i-j}\right) \\
\times \frac{q^{\left(\begin{array}{c}
n+1 \\
3
\end{array}\right)+(1+A)\left(\begin{array}{c}
n+1 \\
2
\end{array}\right)}}{(q ; q)_{n}^{n+1}} \prod_{k=0}^{n}\left[\begin{array}{c}
A \\
X_{k}
\end{array}\right]\left[\begin{array}{c}
A+n-X_{k} \\
n
\end{array}\right]^{-1} .
\end{array}
$$

2.3 Reformulating the $q$-binomial coefficient in terms of shifted factorials

$$
\left[\begin{array}{c}
A+X_{i}-j \\
X_{i}-j
\end{array}\right]=q^{-A j}\left[\begin{array}{c}
A+X_{i} \\
A
\end{array}\right] \frac{\left(q^{-X_{i}} ; q\right)_{j}}{\left(q^{-A-X_{i}} ; q\right)_{j}}
$$

we obtain the following determinant evaluation formula

$$
\begin{aligned}
\operatorname{det}_{0 \leq i, j \leq n}[ & {\left.\left[\begin{array}{c}
A+X_{i}-j \\
X_{i}-j
\end{array}\right]\right]=\prod_{0 \leq i<j \leq n}\left(q^{-X_{j}}-q^{-X_{i}}\right)\left(1-q^{1+A+i-j}\right) } \\
& \times \frac{q^{2\left(\begin{array}{c}
n+1 \\
3
\end{array}\right)-2 A\left(\begin{array}{c}
n+1 \\
2
\end{array}\right)}}{(q ; q)_{n}^{n+1}} \prod_{k=0}^{n}\left[\begin{array}{c}
A+X_{k} \\
A
\end{array}\right]\left[\begin{array}{c}
-1-A+n-X_{k} \\
n
\end{array}\right]^{-1} .
\end{aligned}
$$

2.4 Applying the $q$-binomial relation

$$
\left[\begin{array}{c}
X_{i}+j \\
A
\end{array}\right]=\left[\begin{array}{c}
X_{i} \\
A
\end{array}\right] \frac{\left(q^{1+X_{i}} ; q\right)_{j}}{\left(q^{1-A+X_{i}} ; q\right)_{j}}
$$


we find the corresponding determinant formula

$$
\begin{aligned}
\operatorname{det}_{0 \leq i, j \leq n}\left[\left[\begin{array}{c}
X_{i}+j \\
A
\end{array}\right]\right] & =\prod_{0 \leq i<j \leq n}\left(q^{X_{j}}-q^{X_{i}}\right)\left(1-q^{1+A+i-j}\right) \\
& \times \frac{q^{2\left(\begin{array}{c}
n+1 \\
3
\end{array}\right)+(1-A)\left(\begin{array}{c}
n+1 \\
2
\end{array}\right)}}{(q ; q)_{n}^{n+1}} \prod_{k=0}^{n}\left[\begin{array}{c}
X_{k} \\
A
\end{array}\right]\left[\begin{array}{c}
X_{k}-A+n \\
n
\end{array}\right]^{-1} .
\end{aligned}
$$

2.5 Observing the $q$-binomial relation

$$
\left[\begin{array}{c}
A \\
X_{i}+j
\end{array}\right]=(-1)^{j} q^{\left(A-X_{i}\right) j-\left({ }_{2}^{j}\right)}\left[\begin{array}{c}
A \\
X_{i}
\end{array}\right] \frac{\left(q^{-A+X_{i}} ; q\right)_{j}}{\left(q^{1+X_{i}} ; q\right)_{j}}
$$

we recover the determinant identity due to Carlitz [4] (cf. Chu [5] also)

$$
\begin{aligned}
& \operatorname{det}_{0 \leq i, j \leq n}\left[q^{j X_{i}}\left[\begin{array}{c}
A \\
X_{i}+j
\end{array}\right]\right]=\prod_{0 \leq i<j \leq n}\left(q^{X_{i}}-q^{X_{j}}\right)\left(1-q^{-A+i-j}\right) \\
& \times \frac{q^{\left(\begin{array}{c}
n+1 \\
3
\end{array}\right)+(1+A)\left(\begin{array}{c}
n+1 \\
2
\end{array}\right)}}{(q ; q)_{n}^{n+1}} \prod_{k=0}^{n}\left[\begin{array}{c}
A \\
X_{k}
\end{array}\right]\left[\begin{array}{c}
X_{k}+n \\
n
\end{array}\right]^{-1}
\end{aligned}
$$

2.6 By invoking the $q$-binomial relation

$$
\left[\begin{array}{c}
A+X_{i}+j \\
X_{i}+j
\end{array}\right]=\left[\begin{array}{c}
A+X_{i} \\
A
\end{array}\right] \frac{\left(q^{1+A+X_{i}} ; q\right)_{j}}{\left(q^{1+X_{i}} ; q\right)_{j}}
$$

we recover another determinant identity due to Carlitz [4] (see Menon [9] also)

$$
\begin{aligned}
\operatorname{det}_{0 \leq i, j \leq n}\left[\left[\begin{array}{c}
A+X_{i}+j \\
X_{i}+j
\end{array}\right]\right] & =\prod_{0 \leq i<j \leq n}\left(q^{X_{j}}-q^{X_{i}}\right)\left(1-q^{1+A+i-j}\right) \\
& \times \frac{q^{2\left(\begin{array}{c}
n+1 \\
3
\end{array}\right)+\left(\begin{array}{c}
n+1 \\
2
\end{array}\right)}}{(q ; q)_{n}^{n+1}} \prod_{k=0}^{n}\left[\begin{array}{c}
A+X_{k} \\
A
\end{array}\right]\left[\begin{array}{c}
X_{k}+n \\
n
\end{array}\right]^{-1}
\end{aligned}
$$

which reduces, for $q \rightarrow 1$, to the binomial determinant of Ostrowski [10].

Furthermore for $\delta=0,1$, we can show the following determinant identity

$$
\begin{aligned}
\operatorname{det}_{0 \leq i, j \leq n}\left[C_{X_{i}+j}^{(\delta)}(q)\right] & =(2 q)^{(1+n)(1+n+\delta)+2 \sum_{l=0}^{n} X_{l}} q^{n(n+1)(1+2 n+6 \delta) / 6} \\
& \times \prod_{k=0}^{n} \frac{\left(q ; q^{2}\right)_{1+k}\left(q ; q^{2}\right)_{\delta+X_{k}}}{\left(q^{2} ; q^{2}\right)_{1+\delta+X_{k}+n}} \prod_{0 \leq i<j \leq n}\left(q^{2 X_{i}}-q^{2 X_{j}}\right)
\end{aligned}
$$

where the $q$-Catalan numbers due to Andrews [2] has been slightly extended by

$$
C_{n}^{(\delta)}(q):=\frac{(2 q)^{1+\delta+2 n}}{1-q^{2+2 \delta+2 n}}\left[\begin{array}{c}
\delta+2 n \\
n
\end{array}\right] \frac{1-q}{(-q ; q)_{n}(-q ; q)_{\delta+n}} .
$$


When $x_{k}=k+\ell$, we get the following Hankel determinant identity

$$
\begin{aligned}
\operatorname{det}_{0 \leq i, j \leq n}\left[C_{i+j+\ell}^{(\delta)}(q)\right] & =(2 q)^{(1+n)(1+\delta+2 n+2 \ell)} q^{n(n+1)(4 n+6 \ell+6 \delta-1) / 6} \\
& \times \prod_{k=0}^{n} \frac{(q ; q)_{1+2 k}\left(q ; q^{2}\right)_{\delta+k+\ell}}{\left(q^{2} ; q^{2}\right)_{1+\delta+k+n+\ell}}
\end{aligned}
$$

Letting $\delta=0$ and $q \rightarrow 1$, we recover further the related results $[1,8,11]$ on the classical Catalan numbers $C_{n}=\frac{1}{n+1}\left(\begin{array}{c}2 n \\ n\end{array}\right)$ :

$$
\operatorname{det}_{0 \leq i, j \leq n}\left[C_{i+j}\right]=1, \quad \operatorname{det}_{0 \leq i, j \leq n}\left[C_{i+j+1}\right]=1, \quad \operatorname{det}_{0 \leq i, j \leq n}\left[C_{i+j+2}\right]=n+2 .
$$

2.7 By means of the $q$-binomial relation

$$
\left[\begin{array}{c}
X_{i}+Y_{j} \\
j
\end{array}\right]\left[\begin{array}{c}
A+X_{i} \\
j
\end{array}\right]^{-1}=q^{\left(Y_{j}-A\right) j} \frac{\left(q^{-X_{i}-Y_{j}} ; q\right)_{j}}{\left(q^{-A-X_{i}} ; q\right)_{j}}
$$

we get the following determinant identity

$$
\begin{array}{r}
\operatorname{det}_{0 \leq i, j \leq n}\left[\left[\begin{array}{c}
X_{i}+Y_{j} \\
j
\end{array}\right]\left[\begin{array}{c}
A+X_{i} \\
j
\end{array}\right]^{-1}\right]=\frac{q^{2\left(\begin{array}{c}
n+1 \\
3
\end{array}\right)-\sum_{k=0}^{n}\left(2 k A+n X_{k}-k Y_{k}\right)}}{(q ; q)_{n}^{n+1} \prod_{k=0}^{n}\left[\begin{array}{c}
n-1-A-X_{k} \\
n
\end{array}\right]} \\
\times \prod_{0 \leq i<j \leq n}\left(q^{X_{i}}-q^{X_{j}}\right)\left(1-q^{1+A-Y_{j}+i-j}\right) .
\end{array}
$$

2.8 In view of the $q$-binomial relation

$$
\left[\begin{array}{c}
X_{i}+Y_{j}+j \\
Y_{j}
\end{array}\right]\left[\begin{array}{c}
X_{i}+Y_{j} \\
Y_{j}
\end{array}\right]^{-1}=\frac{\left(q^{1+X_{i}+Y_{j}} ; q\right)_{j}}{\left(q^{1+X_{i}} ; q\right)_{j}}
$$

we obtain the corresponding determinant formula

$$
\begin{aligned}
& \operatorname{det}_{0 \leq i, j \leq n}\left[\left[\begin{array}{c}
X_{i}+Y_{j}+j \\
Y_{j}
\end{array}\right]\left[\begin{array}{c}
X_{i}+Y_{j} \\
Y_{j}
\end{array}\right]^{-1}\right]=\frac{q^{2\left(\begin{array}{c}
n+1 \\
3
\end{array}\right)+\left(\begin{array}{c}
n+1 \\
2
\end{array}\right)}}{(q ; q)_{n}^{n+1}} \prod_{k=0}^{n}\left[\begin{array}{c}
X_{k}+n \\
n
\end{array}\right]^{-1} \\
& \times \prod_{0 \leq i<j \leq n}\left(q^{X_{j}}-q^{X_{i}}\right)\left(1-q^{1+Y_{j}+i-j}\right) .
\end{aligned}
$$

2.9 According to the $q$-binomial relation

$$
\left[\begin{array}{c}
A+X_{i}+Y_{j} \\
j
\end{array}\right]\left[\begin{array}{c}
X_{i}+j \\
j
\end{array}\right]^{-1}=\frac{\left(q^{1+A+X_{i}+Y_{j}-j} ; q\right)_{j}}{\left(q^{1+X_{i}} ; q\right)_{j}}
$$


we derive the corresponding determinant identity

$$
\begin{aligned}
& \operatorname{det}_{0 \leq i, j \leq n}\left[\left[\begin{array}{c}
A+X_{i}+Y_{j} \\
j
\end{array}\right]\left[\begin{array}{c}
X_{i}+j \\
j
\end{array}\right]^{-1}\right]=\frac{q^{2\left(\begin{array}{c}
n+1 \\
3
\end{array}\right)+\left(\begin{array}{c}
n+1 \\
2
\end{array}\right)}}{(q ; q)_{n}^{n+1}} \prod_{k=0}^{n}\left[\begin{array}{c}
X_{k}+n \\
n
\end{array}\right]^{-1} \\
& \times \prod_{0 \leq i<j \leq n}\left(q^{X_{j}}-q^{X_{i}}\right)\left(1-q^{1+A+Y_{j}+i-2 j}\right) .
\end{aligned}
$$

2.10 Similarly, the $q$-binomial relation

$$
\left[\begin{array}{c}
X_{i}+Y_{j} \\
j
\end{array}\right]\left[\begin{array}{c}
A+X_{i}-j \\
n-j
\end{array}\right]=q^{\left(Y_{j}-A\right) j}\left[\begin{array}{c}
n \\
j
\end{array}\right]\left[\begin{array}{c}
A+X_{i} \\
n
\end{array}\right] \frac{\left(q^{-X_{i}-Y_{j}} ; q\right)_{j}}{\left(q^{-A-X_{i}} ; q\right)_{j}}
$$

leads us to the following binomial determinant evaluation formulae

$$
\begin{aligned}
& \operatorname{det}_{0 \leq i, j \leq n}\left[\left[\begin{array}{c}
X_{i}+Y_{j} \\
j
\end{array}\right]\left[\begin{array}{c}
A+X_{i}-j \\
n-j
\end{array}\right]\right]=\prod_{0 \leq i<j \leq n}\left(q^{-X_{j}}-q^{-X_{i}}\right)\left(1-q^{1+A+i-j-Y_{j}}\right) \\
& \times \frac{q^{\sum_{k=0}^{n}\left(k-1-2 A+Y_{k}\right) k}}{(q ; q)_{n}^{n+1}} \prod_{k=0}^{n} \frac{\left[\begin{array}{c}
n \\
k
\end{array}\right]\left[\begin{array}{c}
A+X_{k} \\
n
\end{array}\right]}{\left[\begin{array}{c}
-1-A+n-X_{k} \\
n
\end{array}\right]}, \\
& \operatorname{det}_{0 \leq i, j \leq n}\left[\left[\begin{array}{c}
X_{i}+j \\
j
\end{array}\right]\left[\begin{array}{c}
A+X_{i}+Y_{j} \\
n-j
\end{array}\right]\right]=\prod_{0 \leq i<j \leq n}\left(q^{-X_{i}}-q^{-X_{j}}\right)\left(1-q^{1+n-A-Y_{n-j}+i-j}\right) \\
& \times \frac{q^{\sum_{k=0}^{n}\left(k-1+A-2 n+Y_{n-k}\right) k}}{(q ; q)_{n}^{n+1}} \prod_{k=0}^{n} \frac{\left[\begin{array}{c}
n \\
k
\end{array}\right]\left[\begin{array}{c}
n+X_{k} \\
n
\end{array}\right]}{\left[\begin{array}{c}
-1-X_{k} \\
n
\end{array}\right]} ;
\end{aligned}
$$

where the last identity is derived from the first one under substitution $j \rightarrow n-j$ on the column index.

\section{Duplicate Determinant Identities}

Performing the parameter replacements in Proposition 2

$$
\begin{aligned}
x_{k} & \rightarrow a x_{k}+c / x_{k} \\
\gamma_{k} & \rightarrow d \gamma_{k} /\left(1+a c d^{2} \gamma_{k}^{2}\right), \\
\alpha_{i j} & \rightarrow b \alpha_{i j} /\left(1+a b^{2} c \alpha_{i j}^{2}\right)
\end{aligned}
$$


and then applying factorizations

$$
\begin{aligned}
x_{i}-x_{j} & \rightarrow\left(x_{i}-x_{j}\right)\left(a-c / x_{i} x_{j}\right) \\
\alpha_{i j}-\gamma_{k} & \rightarrow \frac{\left(b \alpha_{i j}-d \gamma_{k}\right)\left(1-a b c d \alpha_{i j} \gamma_{k}\right)}{\left(1+a b^{2} c \alpha_{i j}^{2}\right)\left(1+a c d^{2} \gamma_{k}^{2}\right)} \\
1-x_{i} \gamma_{k} & \rightarrow \frac{\left(1-a d \gamma_{k} x_{i}\right)\left(1-c d \gamma_{k} / x_{i}\right)}{1+a c d^{2} \gamma_{k}^{2}} \\
1-x_{k} \alpha_{i j} & \rightarrow \frac{\left(1-a b x_{k} \alpha_{i j}\right)\left(1-b c \alpha_{i j} / x_{k}\right)}{1+a b^{2} c \alpha_{i j}^{2}}
\end{aligned}
$$

we find the following duplicate determinant identity.

Proposition 4. Let $\left\{x_{k}\right\}_{k=0}^{n}$ be distinct complex numbers. Then there holds the following determinant identity:

$$
\begin{aligned}
\operatorname{det}_{0 \leq i, j \leq n}\left[\frac{\left(a b x_{i} \mid \alpha_{j}\right)_{j}\left(b c / x_{i} \mid \alpha_{j}\right)_{j}}{\left(a d x_{i} \mid \gamma\right)_{j}\left(c d / x_{i} \mid \gamma\right)_{j}}\right] & =\prod_{0 \leq i<j \leq n}\left(b \alpha_{i j}-d \gamma_{j-1}\right)\left(1-a b c d \alpha_{i j} \gamma_{j-1}\right) \\
& \times \frac{\prod_{0 \leq i<j \leq n}\left(x_{i}-x_{j}\right)\left(a-c / x_{i} x_{j}\right)}{\prod_{k=0}^{n}\left(a d x_{k} \mid \gamma\right)_{n}\left(c d / x_{k} \mid \gamma\right)_{n}}
\end{aligned}
$$

This identity contains the following three determinant evaluations.

Corollary 5 ( $a=b=1$ and $\gamma_{k} \rightarrow 0$ in Proposition 4).

$$
\operatorname{det}_{0 \leq i, j \leq n}\left[\left(x_{i} \mid \alpha_{j}\right)_{j}\left(c / x_{i} \mid \alpha_{j}\right)_{j}\right]=\prod_{0 \leq i<j \leq n}\left\{\alpha_{i j}\left(x_{i}-x_{j}\right)\left(1-c / x_{i} x_{j}\right)\right\} .
$$

Corollary 6 ( $d=1$ and $\alpha_{i j} \rightarrow 0$ in Proposition 4).

$$
\operatorname{det}_{0 \leq i, j \leq n}\left[\frac{1}{\left(a x_{i} \mid \gamma\right)_{j}\left(c / x_{i} \mid \gamma\right)_{j}}\right]=\frac{\prod_{0 \leq i<j \leq n}\left(x_{j}-x_{i}\right)\left(a-c / x_{i} x_{j}\right)}{\prod_{k=0}^{n}\left(a x_{k} \mid \gamma\right)_{n}\left(c / x_{k} \mid \gamma\right)_{n}} \prod_{\ell=1}^{n} \gamma_{\ell-1}^{\ell} .
$$

Putting $\alpha_{i j}=p^{i} y_{j}$ and $\gamma_{k}=q^{k}$ in Proposition 4, we find the following determinant evaluation formula of factorial fractions with two different bases.

Corollary 7 (Bibasic determinant identity).

$$
\begin{aligned}
\operatorname{det}_{0 \leq i, j \leq n}\left[\frac{\left(a b x_{i} y_{j} ; p\right)_{j}\left(b c y_{j} / x_{i} ; p\right)_{j}}{\left(a d x_{i} ; q\right)_{j}\left(c d / x_{i} ; q\right)_{j}}\right] & =d^{\left(\begin{array}{c}
n+1 \\
2
\end{array}\right)} \prod_{0 \leq i<j \leq n}\left(x_{j}-x_{i}\right)\left(a-c / x_{i} x_{j}\right) \\
& \times q^{2\left(\begin{array}{c}
n+1 \\
3
\end{array}\right)} \prod_{k=0}^{n} \frac{\left(q^{1-k} b y_{k} / d ; p\right)_{k}\left(q^{k-1} a b c d y_{k} ; p\right)_{k}}{\left(a d x_{k} ; q\right)_{n}\left(c d / x_{k} ; q\right)_{n}} .
\end{aligned}
$$


When $p=q$ and $y_{k}=1$, it reduces to the following determinant identity

$$
\begin{aligned}
\operatorname{det}_{0 \leq i, j \leq n}\left[\frac{\left(a b x_{i} ; q\right)_{j}\left(b c / x_{i} ; q\right)_{j}}{\left(a d x_{i} ; q\right)_{j}\left(c d / x_{i} ; q\right)_{j}}\right] & =b^{\left(\begin{array}{c}
n+1 \\
2
\end{array}\right)} \prod_{0 \leq i<j \leq n}\left(x_{i}-x_{j}\right)\left(a-c / x_{i} x_{j}\right) \\
& \times q^{\left(\begin{array}{c}
n+1 \\
3
\end{array}\right)} \prod_{k=0}^{n} \frac{(d / b ; q)_{k}\left(q^{k-1} a b c d ; q\right)_{k}}{\left(a d x_{k} ; q\right)_{n}\left(c d / x_{k} ; q\right)_{n}} .
\end{aligned}
$$

The determinant evaluation formulae established in this section contain numerous $q$-binomial determinant identities as special cases, which will be illustrated by the following five examples.

3.1 Expressing the $q$-binomial coefficients in terms of shifted factorials

$$
\frac{\left[\begin{array}{c}
X_{i}+A \\
j
\end{array}\right]\left[\begin{array}{c}
X_{i}-B-C \\
n-j
\end{array}\right]}{\left[\begin{array}{c}
X_{i}+B \\
j
\end{array}\right]\left[\begin{array}{c}
X_{i}-A-C \\
n-j
\end{array}\right]}=q^{(A-B) j} \frac{\left[\begin{array}{c}
X_{i}-B-C \\
n
\end{array}\right]}{\left[\begin{array}{c}
X_{i}-A-C \\
n
\end{array}\right]} \times \frac{\left(q^{1+X_{i}-A-C-n} ; q\right)_{j}\left(q^{-X_{i}-A} ; q\right)_{j}}{\left(q^{1+X_{i}-B-C-n} ; q\right)_{j}\left(q^{-X_{i}-B} ; q\right)_{j}}
$$

we establish from Corollary 7 the determinant evaluation formula

$$
\begin{aligned}
& \operatorname{det}_{0 \leq i, j \leq n}\left[\frac{\left.\begin{array}{c}
X_{i}+A \\
j
\end{array}\right]\left[\begin{array}{c}
X_{i}-B-C \\
n-j
\end{array}\right]}{\left[\begin{array}{c}
X_{i}+B \\
j
\end{array}\right]\left[\begin{array}{c}
X_{i}-A-C \\
n-j
\end{array}\right]} q^{\left(\begin{array}{c}
j \\
2
\end{array}\right)}\right]=\prod_{0 \leq i<j \leq n}\left(q^{X_{i}}-q^{X_{j}}\right)\left(1-q^{n-1+C-X_{i}-X_{j}}\right) \\
& \times \frac{q^{B\left(\begin{array}{c}
n+1 \\
2
\end{array}\right)}}{(q ; q)_{n}^{n+1}} \prod_{k=0}^{n} \frac{\left[\begin{array}{c}
n+A+B+C-k \\
k
\end{array}\right]\left[\begin{array}{c}
B-A \\
k
\end{array}\right]\left[\begin{array}{c}
X_{k}-B-C \\
n
\end{array}\right]}{\left[\begin{array}{c}
n \\
k
\end{array}\right]\left[\begin{array}{c}
X_{k}+B \\
n
\end{array}\right]\left[\begin{array}{c}
n-1+B+C-X_{k} \\
n
\end{array}\right]\left[\begin{array}{c}
X_{k}-A-C \\
n
\end{array}\right]}
\end{aligned}
$$

which contains, as special case, the following $q$-binomial determinant identity

$$
\begin{aligned}
& \operatorname{det}_{0 \leq i, j \leq n}\left[\frac{\left[\begin{array}{c}
\lambda i+A \\
j
\end{array}\right]\left[\begin{array}{c}
\lambda i-B \\
n-j
\end{array}\right]}{\left[\begin{array}{c}
\lambda i+B \\
j
\end{array}\right]\left[\begin{array}{c}
\lambda i-A \\
n-j
\end{array}\right]} q^{\left(\begin{array}{c}
j \\
2
\end{array}\right)}\right]=\prod_{0 \leq i<j \leq n}\left(q^{\lambda i}-q^{\lambda j}\right)\left(1-q^{n-1-\lambda i-\lambda j}\right) \\
& \times \frac{q^{B\left(\begin{array}{c}
n+1 \\
2
\end{array}\right)}}{(q ; q)_{n}^{n+1}} \prod_{k=0}^{n} \frac{\left[\begin{array}{c}
n+A+B-k \\
k
\end{array}\right]\left[\begin{array}{c}
B-A \\
k
\end{array}\right]\left[\begin{array}{c}
\lambda k-B \\
n
\end{array}\right]}{\left[\begin{array}{c}
n \\
k
\end{array}\right]\left[\begin{array}{c}
\lambda k+B \\
n
\end{array}\right]\left[\begin{array}{c}
n-1+B-\lambda k \\
n
\end{array}\right]\left[\begin{array}{c}
\lambda k-A \\
n
\end{array}\right]} .
\end{aligned}
$$

3.2 Rewriting the $q$-binomial coefficients in terms of shifted factorials

$$
\frac{\left[\begin{array}{c}
X_{i}+Y_{j} \\
j
\end{array}\right]\left[\begin{array}{c}
A-X_{i}+Y_{j} \\
j
\end{array}\right]}{\left[\begin{array}{c}
B+X_{i} \\
j
\end{array}\right]\left[\begin{array}{c}
A+B-X_{i} \\
j
\end{array}\right]}=q^{2 j\left(Y_{j}-B\right)} \frac{\left(q^{-X_{i}-Y_{j}} ; q\right)_{j}\left(q^{X_{i}-A-Y_{j}} ; q\right)_{j}}{\left(q^{-X_{i}-B} ; q\right)_{j}\left(q^{X_{i}-A-B} ; q\right)_{j}}
$$

we recover from Corollary 7 the determinant identity due to Joris Van Jeugt

$$
\begin{aligned}
\operatorname{det}_{0 \leq i, j \leq n}\left[\frac{\left[\begin{array}{c}
X_{i}+Y_{j} \\
j
\end{array}\right]\left[\begin{array}{c}
A-X_{i}+Y_{j} \\
j
\end{array}\right]}{\left[\begin{array}{c}
B+X_{i} \\
j
\end{array}\right]\left[\begin{array}{c}
A+B-X_{i} \\
j
\end{array}\right]} q^{\left(\begin{array}{l}
j \\
2
\end{array}\right)}\right] & =\prod_{0 \leq i<j \leq n}\left(q^{X_{i}}-q^{X_{j}}\right)\left(1-q^{A-X_{i}-X_{j}}\right) \\
& \times \frac{q^{\sum_{k=0}^{n} k Y_{k}}}{(q ; q)_{n}^{n+1}} \prod_{k=0}^{n} \frac{\left[\begin{array}{c}
1+A+B+Y_{k}-k \\
k
\end{array}\right]\left[\begin{array}{c}
B-Y_{k} \\
k
\end{array}\right]}{\left[\begin{array}{c}
n \\
k
\end{array}\right]\left[\begin{array}{c}
B+B-X_{k} \\
n
\end{array}\right]} .
\end{aligned}
$$


This identity can further be specialized to the $q$-binomial determinant evaluation

$$
\begin{aligned}
\operatorname{det}_{0 \leq i, j \leq n}\left[\frac{\left[\begin{array}{c}
A+\lambda i+j \\
j
\end{array}\right]\left[\begin{array}{c}
A-\lambda i+j \\
j
\end{array}\right]}{\left[\begin{array}{c}
B+\lambda i \\
j
\end{array}\right]\left[\begin{array}{c}
B-\lambda i \\
j
\end{array}\right]} q^{\left(\begin{array}{l}
j \\
2
\end{array}\right)}\right] & =\prod_{0 \leq i<j \leq n}\left(q^{-\lambda i}-q^{-\lambda j}\right)\left(1-q^{\lambda i+\lambda j}\right) \\
& \times \frac{q^{\sum_{k=0}^{n} k(A+k)}}{(q ; q)_{n}^{n+1}} \prod_{k=0}^{n} \frac{\left[\begin{array}{c}
1+A+B \\
k
\end{array}\right]\left[\begin{array}{c}
B-A-k \\
k
\end{array}\right]}{\left[\begin{array}{c}
n \\
k
\end{array}\right]\left[\begin{array}{c}
B+\lambda k \\
n
\end{array}\right]\left[\begin{array}{c}
B-\lambda k \\
n
\end{array}\right]} .
\end{aligned}
$$

3.3 Reformulating the $q$-binomial coefficients in terms of shifted factorials

$$
\frac{\left[\begin{array}{c}
X_{i}+Y_{j}+j \\
X_{i}-Y_{j}-A-j
\end{array}\right]}{\left[\begin{array}{c}
X_{i}+Y_{j} \\
X_{i}-Y_{j}-A
\end{array}\right]\left[\begin{array}{c}
X_{i}+C+j \\
X_{i}-A-C-j
\end{array}\right]}=q^{\left(C-Y_{j}\right) j} \frac{\left[\begin{array}{c}
A+2 C+2 j \\
2 C-2 Y_{j}
\end{array}\right]}{\left[\begin{array}{c}
A+2 C \\
A+2 Y_{j}
\end{array}\right]\left[\begin{array}{c}
C+X_{i} \\
A+2 C
\end{array}\right]} \times \frac{\left(q^{1+X_{i}+Y_{j}} ; q\right)_{j}\left(q^{A-X_{i}+Y_{j}} ; q\right)_{j}}{\left(q^{1+X_{i}+C} ; q\right)_{j}\left(q^{A-X_{i}+C} ; q\right)_{j}}
$$

we derive from Corollary 7 the following determinant formula

$$
\begin{gathered}
\operatorname{det}_{0 \leq i, j \leq n}\left[\frac{\left[\begin{array}{c}
X_{i}+Y_{j}+j \\
X_{i}-Y_{j}-A-j
\end{array}\right]}{\left[\begin{array}{c}
X_{i}+Y_{j} \\
X_{i}-Y_{j}-A
\end{array}\right]\left[\begin{array}{c}
X_{i}+C+j \\
X_{i}-A-C-j
\end{array}\right]}\right]=\prod_{0 \leq i<j \leq n}\left(q^{X_{i}}-q^{X_{j}}\right)\left(1-q^{A-1-X_{i}-X_{j}}\right) \\
\times \frac{q^{2\left(\begin{array}{c}
n+2 \\
3
\end{array}\right)+\left(\begin{array}{c}
n+1 \\
2
\end{array}\right)(C-A)+n \sum_{k=0}^{n} X_{k}}}{(q ; q)_{n}^{n+1}\left[\begin{array}{c}
A+2 C+2 n \\
2 n
\end{array}\right]^{n+1}\left[\begin{array}{c}
2 n \\
n
\end{array}\right]^{n+1}} \prod_{k=0}^{n} \frac{\left[\begin{array}{c}
A+2 C+2 k \\
2 C-2 Y_{k}
\end{array}\right]\left[\begin{array}{c}
Y_{k}-C \\
k
\end{array}\right]\left[\begin{array}{c}
-k-A-C-Y_{k} \\
k
\end{array}\right]}{\left[\begin{array}{c}
n \\
k
\end{array}\right]\left[\begin{array}{c}
A+2 C \\
A+2 Y_{k}
\end{array}\right]\left[\begin{array}{c}
X_{k}+C+n \\
A+2 C+2 n
\end{array}\right]}
\end{gathered}
$$

which reduces, for $X_{i}=b i$ and $Y_{j}=0$, to the $q$-binomial determinant identity:

$$
\begin{aligned}
\underset{0 \leq i, j \leq n}{\operatorname{det}}\left[\left[\begin{array}{c}
b i+j \\
2 j
\end{array}\right]\left[\begin{array}{c}
b i+c+j \\
2 c+2 j
\end{array}\right]^{-1}\right]=\prod_{0 \leq i<j \leq n}\left(q^{-b i}-q^{-b j}\right)\left(1-q^{1+b i+b j}\right) \\
\times \frac{q^{\sum_{k=0}^{n} k(n b+c+k)}}{(q ; q)_{n}^{n+1}\left[\begin{array}{c}
2 c+2 n \\
2 n
\end{array}\right]^{n+1}\left[\begin{array}{c}
2 n \\
n
\end{array}\right]^{n+1}} \prod_{k=0}^{n} \frac{\left[\begin{array}{c}
2 c+2 k \\
2 c
\end{array}\right]\left[\begin{array}{c}
-c \\
k
\end{array}\right]\left[\begin{array}{c}
-k-c \\
k
\end{array}\right]}{\left[\begin{array}{l}
n \\
k
\end{array}\right]\left[\begin{array}{c}
b k+c+n \\
2 c+2 n
\end{array}\right]}
\end{aligned}
$$

3.4 According to Corollary 6, the $q$-binomial relation

$$
q^{j X_{i}}\left[\begin{array}{c}
X_{i}+A-j \\
X_{i}+j
\end{array}\right]=\frac{(-1)^{j} q^{\left(\frac{j}{2}\right)-A j}(q ; q)_{X_{i}+A}}{(q ; q)_{X_{i}}(q ; q)_{A-2 j}} \times \frac{1}{\left(q^{1+X_{i}} ; q\right)_{j}\left(q^{-A-X_{i}} ; q\right)_{j}}
$$

yields the determinant evaluation formula

$$
\begin{aligned}
\underset{0 \leq i, j \leq n}{\operatorname{det}}\left[q^{j X_{i}}\left[\begin{array}{c}
X_{i}+A-j \\
X_{i}+j
\end{array}\right]\right] & =\prod_{0 \leq i<j \leq n}\left(q^{-X_{i}}-q^{-X_{j}}\right)\left(1-q^{1+A+X_{i}+X_{j}}\right) \\
& \times \prod_{k=0}^{n}\left[\begin{array}{c}
X_{k}+A-n \\
X_{k}+n
\end{array}\right] \frac{q^{n X_{k}}}{\left(q^{1+A-2 n} ; q\right)_{2 k}} .
\end{aligned}
$$


In particular for $X_{i}=c+b i$, it becomes the $q$-binomial determinant identity

$$
\begin{array}{r}
\underset{0 \leq i, j \leq n}{\operatorname{det}}\left[q^{b i j}\left[\begin{array}{l}
a+b i-j \\
c+b i+j
\end{array}\right]\right] \\
\times \prod_{0 \leq i<j \leq n}\left(q^{-b i}-q^{-b j}\right)\left(1-q^{1+a+c+b i+b j}\right) \\
\times q^{n b\left(\begin{array}{c}
n+1 \\
2
\end{array}\right)} \prod_{k=0}^{n} \frac{(q ; q)_{a+b k-n}}{(q ; q)_{a-c-2 k}(q ; q)_{c+b k+n}}
\end{array}
$$

which is the $q$-analogue of the determinant evaluated by Amdeberhan and Zeilberger [3, Eq 2].

3.5 In view of Corollary 5 , the $q$-binomial relation

$$
q^{-j X_{i}}\left[\begin{array}{c}
X_{i}+Y_{j}+j \\
X_{i}-Y_{j}+A-j
\end{array}\right]\left[\begin{array}{c}
X_{i}-Y_{j}+A \\
X_{i}+Y_{j}
\end{array}\right]=\frac{(-1)^{j} q^{\left(A-Y_{j}\right) j-\left(\frac{j}{2}\right)}}{(q ; q)_{2 Y_{j}-A+2 j}(q ; q)_{A-2 Y_{j}}} \times\left(q^{1+X_{i}+Y_{j}} ; q\right)_{j}\left(q^{Y_{j}-X_{i}-A} ; q\right)_{j}
$$

leads to the following determinant evaluation

$$
\begin{aligned}
& \underset{0 \leq i, j \leq n}{\operatorname{det}}\left[q^{-j X_{i}}\left[\begin{array}{c}
X_{i}+Y_{j}+j \\
X_{i}-Y_{j}+A-j
\end{array}\right]\left[\begin{array}{c}
X_{i}-Y_{j}+A \\
X_{i}+Y_{j}
\end{array}\right]\right] \\
& =\frac{\prod_{0 \leq i<j \leq n}\left(q^{-X_{j}}-q^{-X_{i}}\right)\left(1-q^{1+A+X_{i}+X_{j}}\right)}{\prod_{k=0}^{n}(q ; q)_{A-2 Y_{k}}(q ; q)_{2 Y_{k}-A+2 k}} .
\end{aligned}
$$

In particular for $X_{i}=a+b i$ and $Y_{j}=0$, the last identity gives

$$
\operatorname{det}_{0 \leq i, j \leq n}\left[q^{-b i j}\left[\begin{array}{l}
a+b i+j \\
c+b i-j
\end{array}\right]\right]=\frac{\prod_{0 \leq i<j \leq n}\left(q^{-b j}-q^{-b i}\right)\left(1-q^{1+a+c+b i+b j}\right)}{\prod_{k=0}^{n}\left(q^{1+a+b k} ; q\right)_{c-a}(q ; q)_{a-c+2 k}}
$$

which results in the $q$-analogue of the binomial determinant identity due to Amdeberhan and Zeilberger [3, Eq 1].

Similarly, letting $x_{i}=q^{a+b i}$ and $\alpha_{i j}=q^{d j-i}$, we find from Corollary 5 another determinant identity

$$
\begin{aligned}
& \operatorname{det}_{0 \leq i, j \leq n}\left[\left[\begin{array}{c}
a+b i+d j \\
j
\end{array}\right]\left[\begin{array}{c}
c-b i+d j \\
j
\end{array}\right] q^{\left(\begin{array}{c}
j \\
2
\end{array}\right)}\right] \\
& =\prod_{k=0}^{n} \frac{q^{k(c+d k)}}{(q ; q)_{k}^{2}} \prod_{0 \leq i<j \leq n}\left(q^{-b i}-q^{-b j}\right)\left(1-q^{a-c+b i+b j}\right)
\end{aligned}
$$

which is the $q$-analogue of the result in Amdeberhan and Zeilberger [3, Eq 14]. The list of examples can be endless. However, we are not going further to prolong it due to the space limitation. 


\section{References}

[1] Aigner, M., Catalan-like numbers and determinants, J. Combin. Theory (Ser. A) 87 (1999), 33-51.

[2] Andrews, G.E., Catalan numbers, $q$-Catalan numbers and hypergeometric series, J. Combin. Theory (Ser. A) 44 (1987), 267-273.

[3] Amdeberhan, T. And Zeilberger, D., Determinants through the looking glass, Adv. in Appl. Math. 27 (2001), 225-230.

[4] Carlitz, L., Some determinants of $q$-binomial coefficients, J. Reine Angew. Math. 226 (1967), 216-220.

[5] CHU, W., On the evaluation of some determinants with $q$-binomial coefficients, J. Systems Science \& Math. Science 8:4 (1988), 361-366.

[6] Chu, W., Generalizations of the Cauchy determinant, Publicationes Mathematicae Debrecen 58:3 (2001), 353-365.

[7] Chu, W., The Cauchy double alternant and divided differences, Electronic Journal of Linear Algebra 15 (2006), 14-21.

[8] CigleR, J., Operatormethoden für q-Identitäten. VII: $q$-Catalan-Determinanten, Österreich. Akad. Wiss. Math.-Natur. Kl. Sitzungsber. II 208 (1999), 123-142.

[9] Menon, K.V., Note on some determinants of $q$-binomial numbers, Discrete Math. 61:2-3 (1986), 337-341.

[10] Ostrowski, A.M., On some determinants with combinatorial numbers, J. Reine Angew. Math. 216 (1964), 25-30.

[11] Radoux, C., Nombres de Catalan généralisés, Bull. Belg. Math. Soc. Simon Stevin 4:2 (1997), 289-292. 